ఠ

\title{
Anti-NMDA receptor antibodies in patients with a first episode of schizophrenia
}

This article was published in the following Dove Press journal:

Neuropsychiatric Disease and Treatment

6 March 2015

Number of times this article has been viewed

\section{Jiří Masopust ${ }^{1,2}$ \\ Ctirad Andrýs ${ }^{3}$ \\ Jan Bažant ${ }^{\prime}$ \\ Oldřich Vyšata ${ }^{4}$ \\ Kamil Kuca ${ }^{5}$ \\ Martin Vališ}

'Department of Psychiatry, Faculty of Medicine in Hradec Kralové, Charles University in Prague, University Hospital Hradec Králové, Hradec Králové, Czech Republic; ${ }^{2}$ National Institute of Mental Health, Klecany, Czech Republic; ${ }^{3}$ Institute of Clinical Immunology and Allergology, Charles University in Prague, Faculty of Medicine in Hradec Králové, and University Hospital Hradec Králové, Hradec Králové, Czech Republic; ${ }^{4}$ Department of Neurology, Charles University in Prague, Faculty of Medicine in Hradec Králové, and University Hospital Hradec Králové, Hradec Králové, Czech Republic; ${ }^{5}$ Biomedical Research Centrum, University Hospital Hradec Králové, Hradec Králové, Czech Republic
Correspondence: Martin Vališ Department of Neurology, University Hospital, Sokolská 58I, Hradec Králové, 50005 Czech Republic

Tel +420 49583525 I

Fax +420 495835200

Email valismar@seznam.cz

\begin{abstract}
Background: Encephalitis with antibodies against N-methyl-D-aspartate receptor (NMDA-R) is classified as an autoimmune disorder with psychotic symptoms, which are frequently dominant. However, it remains unclear how frequently NMDA-R antibodies lead to a condition that mimics psychosis and first-episode schizophrenia. In our work, we investigated the presence of antibodies against NMDA-R in patients with first-episode psychosis (FEP) in comparison with healthy volunteers.
\end{abstract}

Methods: This study included 50 antipsychotic-naïve patients with FEP (including 21 women) and 50 healthy volunteers (including 21 women). The mean age of the patients was $27.4( \pm 7.4)$ years and that of the healthy controls was $27.0( \pm 7.3)$ years. Antibodies against NMDA-R in the serum were detected by immunofluorescence.

Results: None of the investigated patients with an FEP and none of the healthy controls showed positive antibodies against NMDA-Rs.

Conclusion: According to results of studies, a small proportion of patients with an FEP possess antibodies against NMDA-R. However, the extent to which this finding contributes to the etiopathogenesis of the response to antipsychotic medication and whether immunomodulatory therapy is indicated in these cases remains uncertain.

Keywords: N-methyl-D-aspartate receptor, NMDA-R, encephalitis, schizophrenia

\section{Introduction}

Encephalitis cases positive for antibodies against N-methyl-D-aspartate receptor (NMDA-R) show clinical features consistent with autoimmune disease, with a predominance of psychiatric symptoms. This condition was first described in 2007 in a group of 12 women with ovarian teratomas who suffered from a paraneoplastic syndrome. ${ }^{1}$ All forms of anti-NMDA-R encephalitis are associated with the failure to detect tumor tissue and present a unique type of autoimmune reaction. ${ }^{2}$ This disease is much more common in women ( $80 \%$ ) and younger individuals (median 23 years), although it may occur at any age. ${ }^{3}$ In addition, a large number of cases are unrelated to cancer. ${ }^{4}$

In most cases, the first symptoms of the disease are behavioral and psychiatric, including psychosis, anxiety, insomnia, mania, and catatonic symptoms. ${ }^{4,5}$ These symptoms typically develop very rapidly, and a few days before their development, nonspecific "flu-like" symptoms may be present. Short-term memory disturbances, speech disorders, delirium, seizures, and extrapyramidal symptomatology characteristic of autonomic dysfunction, in the form of hypotension, heart rhythm disorders, hyperthermia, and central hypoventilation requiring complex intensive care, including artificial lung ventilation, may also develop. Milder cases may present with psychiatric symptomatology as well as memory disorders, epileptic seizures, or dyskinesia. ${ }^{2}$ The underlying mechanism for this disease is the binding of immunoglobulin (Ig)G1 and IgG3 antibodies to the NR1 subunit of NMDA-R. The diagnosis of anti-NMDA-R 
encephalitis is based on the detection of specific IgG autoantibodies from serum and cerebrospinal fluid (CSF) samples. The sensitivity of detection is higher in CSF samples compared with serum samples (up to $100 \%$ of patients are positive for NMDA-R antibodies in the CSF compared with the $85 \%$ of patients who are positive for antibodies in the serum). ${ }^{2}$ The specificity of antibody detection is very high; however, in individual cases, positivity of serum antibodies has been recorded in other diseases (schizophrenia, narcolepsy, and herpes encephalitis). ${ }^{6,7}$

Antibodies against NMDA-R may be associated with psychoses for several reasons. Psychotic symptoms are observed in $65 \%$ of encephalitis cases with antibodies against NMDA-R. ${ }^{8}$ Immune and autoimmune abnormalities are also found in patients with schizophrenia, although these findings are inconsistent and have no practical use in the diagnosis and treatment of schizophrenia. ${ }^{5}$ Dysfunction of NMDA-R and the glutamatergic system may be associated with the pathogenesis of schizophrenia, as antagonists of NMDA-R, including phencyclidine and ketamine, have been shown to induce psychotic symptoms (positive and negative) and behavioral and cognitive impairments similar to those observed in patients with schizophrenia. ${ }^{9,10}$ Furthermore, the administration of ketamine, as well as CSF containing anti-NMDA-R antibodies, has been shown to increase the extracellular glutamate level in animals. ${ }^{11,12}$

The occurrence of antibodies against NMDA-R in patients with schizophrenia has been the focus of seven studies, ${ }^{6,13-18}$ which were included in a recent meta-analysis. ${ }^{5}$ Of the 1,441 patients included in this analysis, $115(7.98 \%)$ (95\% confidence interval [CI] 6.69 to 9.50 ) patients tested positive for antibodies against NMDA-R; of these patients, $21(1.46 \%)(95 \%$ CI 0.94 to 2.23$)$ presented antibodies of the IgG subclass. Of the 272 patients with first-episode psychosis (FEP), 14 tested positive for anti-NMDA-R antibodies (5.15\%) (95\% CI 3.02 to 8.52), and five cases showed antibodies of the IgG subclass.
In the current work, we investigated the presence of antibodies against NMDA-R in patients with FEP in comparison with corresponding healthy volunteers.

\section{Methods}

\section{Subjects}

This study included 50 patients with FEP and 50 healthy volunteers of comparable age and sex. The demographic and clinical data are presented in Table 1.

The patients were recruited for the study in the Department of Psychiatry of the University Hospital in Hradec Králové. The inclusion criteria were as follows: hospitalized patients with FEP (schizophrenia F20 and acute schizophreniform psychosis F23.2, according to the ICD-10 classification); between 18-55 years of age; antipsychotic-naïve; and without serious medical comorbidities. We excluded patients with preexisting cardiovascular, pulmonary, or neurological diseases by reviewing patient medical records. We conducted a comprehensive physical and laboratory assessment, which was supplemented with patient family history. Healthy volunteers were recruited from the staff at the University Hospital in Hradec Králové. Healthy volunteers without any mental or serious somatic disorder were matched to the patient sample with respect to age and sex. The possibility of mental illness among the volunteers was excluded by a psychiatric examination, performed by a psychiatrist. All of the participants voluntarily signed an informed consent form.

\section{Procedures}

Venous blood from the patients and healthy volunteers was obtained between 7 and 9 am, after 12 hours of fasting. The coagulated blood samples were centrifuged to separate the blood elements from the serum. The serum was stored in aliquots at $-70^{\circ} \mathrm{C}$ prior to analysis.

NMDA-R autoantibodies were detected, using a commercial indirect immunofluorescence assay, with a Neurology Mosaics Test (Euroimmun AG, Lübeck, Germany).

Table I Demographic and clinical data of the patients and healthy volunteers

\begin{tabular}{|c|c|c|c|c|c|c|c|}
\hline & \multirow{2}{*}{\multicolumn{3}{|c|}{$\begin{array}{l}\text { Patients }(n=50) \\
\text { Women }(n=2 I)\end{array}$}} & \multirow{2}{*}{\multicolumn{3}{|c|}{$\begin{array}{l}\text { Healthy volunteers }(n=50) \\
\text { Women }(n=2 I)\end{array}$}} & \multirow[t]{3}{*}{$P$ (test) } \\
\hline & & & & & & & \\
\hline & Mean (SD) & Median & Range & Mean (SD) & Median & Range & \\
\hline Age (years) & $27.4(7.4)$ & 27 & $18-52$ & $27.0(7.3)$ & 26 & $18-53$ & NS (MW-U) \\
\hline Weight (kg) & $66.9(13.1)$ & 67.5 & $39-102$ & $74.1(14.2)$ & 71.5 & $51.6-109$ & $0.02(\mathrm{MW}-U)$ \\
\hline $\mathrm{BMI}$ & $22.4(4.2)$ & 22.0 & $13.4-40$ & $23.7(3.0)$ & 23.9 & $18.4-30.9$ & $0.02(\mathrm{MW}-U)$ \\
\hline Duration of illness (months) & $8.2(14.1)$ & I & $0.25-60$ & - & - & - & - \\
\hline PANSS positive scale & $25.5(6.0)$ & 25 & $16-39$ & - & - & - & - \\
\hline PANSS total score & $97.7(16.6)$ & 97.5 & $62-145$ & - & - & - & - \\
\hline
\end{tabular}

Abbreviations: BMI, body mass index; MW-U, Mann-Whitney U-test; PANSS, Positive and Negative Syndrome Scale; SD, standard deviation; NS, not significant. 
Autoantibodies against glutamate receptors of the NMDA type show the typical fluorescence of transfected cells. All samples were classified as positive or negative for NMDA-R antibodies (Figure 1).

Psychopathology was assessed using the Positive and Negative Syndrome Scale (PANSS). ${ }^{19}$ We also measured the body weight and height of the subjects to calculate the body mass index (BMI).

\section{Statistical analysis}

We used Statistica 8 statistical software to test for differences between the patients and healthy volunteers (for age, weight, and BMI). The statistical evaluation was conducted with nonparametric methods, specifically, the Mann-Whitney $U$-test. All of the tests were two-tailed, with $\alpha<0.05$.

\section{Ethical aspects}

The details of this study were approved by the Ethical Committee of the University Hospital in Hradec Králové. Even when the patients had signed the written informed consent in a state of acute psychosis, they later agreed to continue in the study during remission.

\section{Results}

A total of 50 patients with FEP and 50 age-and sex-matched healthy volunteers were analyzed. Neither the patients with an FEP or the healthy controls showed positive antibodies against NMDA-R. The healthy volunteers showed significantly greater weight and BMI (both $P=0.02$ ).

\section{Discussion}

NMDA-Rs are ionotropic glutamate receptors that allow excitatory neurotransmission and contribute to the plasticity
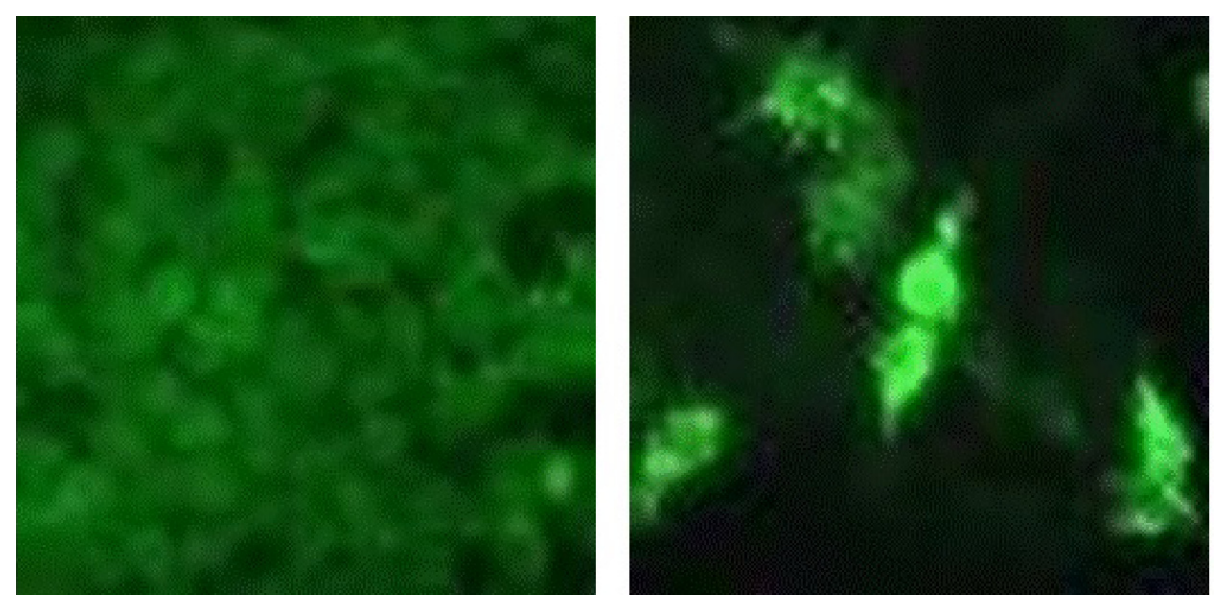

of the brain and to learning and memory processes, through specific long-term potentiation of neuronal connections. These receptors consist of two subunits, NR1 and NR2, which exist in several isoforms with differential expression throughout the brain and during development. ${ }^{20}$ The highest density of receptors is found in the amygdala, prefrontal cortex, hippocampus and hypothalamus. Reduced NMDA-R activity can lead to the development of psychotic symptoms. Autoantibodies against NMDA-R may be produced in the context of cross-reactivity against the tumor cells of teratomas, which contain heterogeneous cell types, including nerve cells. Autoantibodies are directed specifically against the NR1A subunit, and receptor binding leads to internalization of the NMDA-R. However, the reduction in the number of surface receptors is reversible and depends on the antibody titer. $^{2}$ Schizophrenia includes a heterogeneous group of disorders. The catatonic symptoms of schizophrenia may be related to glutamatergic dysfunction, and the use of the noncompetitive NMDA antagonist memantine has been suggested for the treatment of catatonia. ${ }^{21}$ In schizophrenic patients resistant to treatment, normal findings of dopaminergic neurotransmission in brain imaging could be presumed to result from autoimmune dysfunction in a conditional glutamatergic system. ${ }^{5}$

In previous studies, positivity for antibodies against NMDA-R was investigated in 1,441 patients with psychosis and 1,598 healthy controls, although the occurrence of any subtype of these antibodies (IgG, IgM, or IgA) was significantly higher in healthy volunteers compared with patients with FEP $(P=0.003) .{ }^{5}$ Of the 144 healthy volunteers with positive subtype antibodies, 143 were from the study of Hammer et al. ${ }^{18}$ Although it remains unclear whether the results would have been the same if age-matched controls were included,

Figure I Left negative and right positive result for NMDA-R antibodies. 
the IgG subtype was significantly more frequent in patients with FEP than in the control group $(P<0.01)$. There was heterogeneity between studies in terms of the assay used to establish the presence of the autoantibodies. The size of the study samples were different. Methodologically, the most sophisticated study was that of Steiner et $\mathrm{al}^{17}$ who used a prospective study design, had both psychiatric and nonpsychiatric control groups, and looked at multiple Ig subtypes aimed at different NMDA-R epitopes. ${ }^{5}$

Our study of 50 patients contributes to the findings of a previous study, which included 272 patients with FEP. ${ }^{5}$ Although we did not detect antibodies against NMDA-R in the serum of any of the patients with FEB or healthy controls, identical results were obtained, in the study by Masdeu et $\mathrm{al}^{16}$ in a group of 80 patients with FEP and 40 controls. In addition, the absence of NMDA-R antibodies was also reported in studies by Rhoads et $\mathrm{al}^{14}$ and Haussleiter et al ${ }^{15}$ who enrolled patients undergoing long-term treatment for psychosis. In contrast, Zandi et $\mathrm{al}^{13}$ found positive results in $6.52 \%$ of 46 FEP patients $(n=3)$, and one patient improved after immunotherapy. In a study by Tsutsui et $\mathrm{al}^{6}$ of 51 patients with schizophrenia or schizoaffective disorder, there were four positive findings. In addition, Steiner et $\mathrm{al}^{17}$ examined the prevalence of antibodies against NMDA-R in serum samples from 121 patients initially diagnosed with schizophrenia, and NR1 IgG antibodies were detected in four patients, two of whom presented retrospectively with overdiagnosed encephalitis and antibodies against NMDA-R. However, one discrepant study ${ }^{18}$ yielded positive findings for IgG antibodies in seven of 1,081 patients with psychosis (including FEP).

Our results may have been affected by the laboratory method used to detect autoantibodies. Indeed, the various methods used in previous studies appear to contribute to the heterogeneity of these results. The recommended three-stage diagnosis ${ }^{22}$ was used in the study of Masdeu et al ${ }^{16}$ although whether antibodies should be measured in the serum or CSF remains controversial. ${ }^{5,11,23}$ Relatively small study sample size may be an additional limitation of our study.

The interpretation of NMDA-R antibody positivity in patients with FEP and the implications for therapeutic procedures remain unclear. Antibody detection could represent a continuum of states ranging from a "clean" psychotic presentation to catatonic symptoms following the full expression of encephalitis, which carries a potentially lethal outcome. The presence of antibodies against NMDA-R in other diseases (bipolar disorder, depression, narcolepsy with psychosis, autism, and Creutzfeld-Jakob disease) highlights the possibility that their presence is caused by neuronal damage or other causes. ${ }^{5}$ In previous analyses, patients with psychotic symptoms who were diagnosed with encephalitis and showed the presence of antibodies against NMDA-R, generally responded well to immunotherapy. ${ }^{13,24}$ Thus, some authors recommend screening for the presence of antibodies against NMDA-R in all patients with FEP. ${ }^{8}$ This possibility should undoubtedly be considered in cases of rapid-onset psychosis, catatonic manifestations, "flu-like" prodromal symptoms, incidence of seizures or other neurological dysfunctions (aphasia), severe autonomic manifestations, or deterioration during the administration of antipsychotics. ${ }^{25}$

\section{Conclusion}

A minority of patients with FEP present with autoantibodies against NMDA-R. However, it remains uncertain to what extent this finding contributes to the etiopathogenesis of psychosis or the response to antipsychotic medication, and it is also unknown whether immunomodulatory therapy should be indicated in these cases.

\section{Acknowledgments}

This study was supported by scientific program of the Czech Ministry of Health - conceptual development of research organization (MH CZ - DRO) (UHHK, 00179906); MH CZ - DRO (PCP, 00023752); Ministry of Education, Youth and Sports - Institutional Support for Longterm Development of Research Organizations - Charles University PRVOUK P37/08 and P37/03 and National Institute of Mental Health (NIMH) CZ.1.05/2.1.00/03.0078 from the European Regional Development Fund.

\section{Disclosure}

The authors report no conflicts of interest in this work.

\section{References}

1. Dalmau J, Tüzün E, Wu HY, et al. Paraneoplastic anti-N-methyl-Daspartate receptor encephalitis associated with ovarian teratoma. Ann Neurol. 2007;61(1):25-36.

2. Dalmau J, Lancaster E, Martinez-Hernandez E, Rosenfeld MR, BaliceGordon R. Clinical experience and laboratory investigations in patients with anti-NMDAR encephalitis. Lancet Neurol. 2011;10(1):63-74.

3. Dalmau J, Gleichman AJ, Hughes EG, et al. Anti-NMDA-receptor encephalitis: case series and analysis of the effects of antibodies. Lancet Neurol. 2008;7(12):1091-1098.

4. Titulaer MJ, McCracken L, Gabilondo I, et al. Treatment and prognostic factors for long-term outcome in patients with anti-NMDA receptor encephalitis: an observational cohort study. Lancet Neurol. 2013;12(2): 157-165.

5. Pollak TA, McCormack R, Peakman M, Nicholson TR, David AS. Prevalence of anti-N-methyl-D-aspartate (NMDA) receptor antibodies in patients with schizophrenia and related psychoses: a systematic review and meta-analysis. Psychol Med. 2014;44(12):2475-2487. 
6. Tsutsui K, Kanbayashi T, Tanaka K, et al. Anti-NMDA-receptor antibody detected in encephalitis, schizophrenia, and narcolepsy with psychotic features. BMC Psychiatry. 2012;12:37.

7. Ezeoke A, Mellor A, Buckley P, Miller B. A systematic, quantitative review of blood autoantibodies in schizophrenia. Schizophr Res. 2013; 150(1):245-251.

8. Lennox BR, Coles AJ, Vincent A. Antibody-mediated encephalitis: a treatable cause of schizophrenia. Br J Psychiatry. 2012;200(2):92-94.

9. Corlett PR, Honey GD, Krystal JH, Fletcher PC. Glutamatergic model psychoses: prediction error, learning, and inference. Neuropsychopharmacology. 2011;36(1):294-315.

10. Javitt DC. Glutamate and schizophrenia: phencyclidine, N-methylD-aspartate receptors, and dopamine-glutamate interactions. Int Rev Neurobiol. 2007;78:69-108.

11. Irani SR, Bera K, Waters P, et al. N-methyl-D-aspartate antibody encephalitis: temporal progression of clinical and paraclinical observations in a predominantly non-paraneoplastic disorder of both sexes. Brain. 2010;133(Pt 6):1655-1667.

12. Manto M, Dalmau J, Didelot A, Rogemond V, Honnorat J. In vivo effects of antibodies from patients with anti-NMDA receptor encephalitis: further evidence of synaptic glutamatergic dysfunction. Orphanet J Rare Dis. 2010;5:31.

13. Zandi MS, Irani SR, Lang B, et al. Disease-relevant autoantibodies in first episode schizophrenia. J Neurol. 2011;258(4):686-688.

14. Rhoads J, Guirgis H, McKnight C, Duchemin AM. Lack of anti-NMDA receptor autoantibodies in the serum of subjects with schizophrenia. Schizophr Res. 2011;129(2-3):213-214.

15. Haussleiter IS, Emons B, Schaub M, et al. Investigation of antibodies against synaptic proteins in a cross-sectional cohort of psychotic patients. Schizophr Res. 2012;140(1-3):258-259.

16. Masdeu JC, González-Pinto A, Matute C, et al. Serum IgG antibodies against the NR1 subunit of the NMDA receptor not detected in schizophrenia. Am J Psychiatry. 2012;169(10):1120-1121.
17. Steiner J, Walter M, Glanz W, et al. Increased prevalence of diverse $\mathrm{N}$-methyl-D-aspartate glutamate receptor antibodies in patients with an initial diagnosis of schizophrenia: specific relevance of IgG NR1a antibodies for distinction from $\mathrm{N}$-methyl-D-aspartate glutamate receptor encephalitis. JAMA Psychiatry. 2013;70(3):271-278.

18. Hammer C, Stepniak B, Schneider A, et al. Neuropsychiatric disease relevance of circulating anti-NMDA receptor autoantibodies depends on blood-brain barrier integrity. Mol Psychiatry. 2014;19(10): 1143-1149.

19. Kay SR. Positive and Negative Symptoms in Schizophrenia. Assessment and Research. New York: Brunner-Mazel;1991.

20. Waxman EA, Lynch DR. N-methyl-D-aspartate receptor subtypes: multiple roles in excitotoxicity and neurological disease. Neuroscientist. 2005;11(1):37-49.

21. Obregon DF, Velasco RM, Wuerz TP, Catalano MC, Catalano G, Kahn D. Memantine and catatonia: a case report and literature review. J Psychiatr Pract. 2011;17(4):292-299.

22. Lancaster E, Dalmau J. Neuronal autoantigens - pathogenesis, associated disorders and antibody testing. Nat Rev Neurol. 2012;8(7):380-390.

23. Pearlman DM, Najjar S. Meta-analysis of the association between N-methyl-D-aspartate receptor antibodies and schizophrenia, schizoaffective disorder, bipolar disorder, and major depressive disorder. Schizophr Res. 2014;157(1-3):249-258.

24. Kayser MS, Titulaer MJ, Gresa-Arribas N, Dalmau J. Frequency and characteristics of isolated psychiatric episodes in anti-N-methyl-D-aspartate receptor encephalitis. JAMA Neurol. 2013;70(9):1133-1139.

25. Maneta E, Garcia G. Psychiatric manifestations of anti-NMDA receptor encephalitis: neurobiological underpinnings and differential diagnostic implications. Psychosomatics. 2014;55(1):37-44.
Neuropsychiatric Disease and Treatment

\section{Publish your work in this journal}

Neuropsychiatric Disease and Treatment is an international, peerreviewed journal of clinical therapeutics and pharmacology focusing on concise rapid reporting of clinical or pre-clinical studies on a range of neuropsychiatric and neurological disorders. This journal is indexed on PubMed Central, the 'PsycINFO' database and CAS,

\section{Dovepress}

and is the official journal of The International Neuropsychiatric Association (INA). The manuscript management system is completely online and includes a very quick and fair peer-review system, which is all easy to use. Visit http://www.dovepress.com/testimonials.php to read real quotes from published authors. 